\title{
Resonant photoluminescence studies of carrier localisation in c-plane InGaN/GaN quantum well structures
}

DOI:

10.1088/1361-648X/aab818

\section{Document Version}

Accepted author manuscript

Link to publication record in Manchester Research Explorer

\section{Citation for published version (APA):}

Blenkhorn, W. E., Schulz, S., Tanner, D. S. P., Oliver, R. A., Kappers, M. J., Humphreys, C. J., \& Dawson, P. (2018). Resonant photoluminescence studies of carrier localisation in c-plane InGaN/GaN quantum well structures. Journal of Physics: Condensed Matter. https://doi.org/10.1088/1361-648X/aab818

\section{Published in:}

Journal of Physics: Condensed Matter

\section{Citing this paper}

Please note that where the full-text provided on Manchester Research Explorer is the Author Accepted Manuscript or Proof version this may differ from the final Published version. If citing, it is advised that you check and use the publisher's definitive version.

\section{General rights}

Copyright and moral rights for the publications made accessible in the Research Explorer are retained by the authors and/or other copyright owners and it is a condition of accessing publications that users recognise and abide by the legal requirements associated with these rights.

\section{Takedown policy}

If you believe that this document breaches copyright please refer to the University of Manchester's Takedown Procedures [http://man.ac.uk/04Y6Bo] or contact uml.scholarlycommunications@manchester.ac.uk providing relevant details, so we can investigate your claim.

\section{OPEN ACCESS}


Resonant photoluminescence studies of carrier localisation in c-plane InGaN/GaN quantum well structures

W. E. Blenkhorn ${ }^{1}$, S. Schulz ${ }^{2}$, D. S. P. Tanner ${ }^{2,3}$, R. A. Oliver ${ }^{4}$, M. J. Kappers ${ }^{4}$, C. J. Humphreys ${ }^{4}$ and P. Dawson ${ }^{1}$

${ }^{1}$ School of Physics and Astronomy, Photon Science Institute, University of Manchester, M13 9PL, Manchester, UK

${ }^{2}$ Photonics Theory Group, Tyndall National Institute, Dyke Parade, Cork, Ireland ${ }^{3}$ Department of Physics, University College Cork, Cork, Ireland

${ }^{4}$ Department of Material Science and Metallurgy, 27 Charles Babbage Road, University of Cambridge, Cambridge, CB3 OFS, UK

\begin{abstract}
In this paper we report on changes in the form of the low temperature $(12 \mathrm{~K})$ photoluminescence spectra of an $\mathrm{InGaN} / \mathrm{GaN}$ quantum well structure as a function of excitation photon energy. As the photon energy is progressively reduced we observe at a critical energy a change in the form of the spectra from one which is determined by the occupation of the complete distribution of hole localisation centres to one which is determined by the resonant excitation of specific localisations sites. This change is governed by an effective mobility edge whereby the photo-excited holes remain localised at their initial energy and are prevented from scattering to other localisation sites. This assignment is confirmed by the results of atomistic tight binding calculations which show that the wave function overlap of the lowest lying localised holes with other hole states is low compared with the overlap of higher lying hole states with other higher lying hole states.
\end{abstract}




\section{Introduction}

The nature of the radiative recombination process(es) in InGaN/GaN quantum wells (QWs) has been extensively investigated since the pioneering work of Akasaki, Amano and Nakamura [1] demonstrated the potential that the $\mathrm{InGaN}$ materials system has for producing optoelectronic devices that emit in the near UV through the visible part of the spectrum. One of the main reasons for this analysis is that the room temperature internal quantum efficiency of light emitting diodes can be very high, despite the presence of extended defects with densities $\sim 10^{8} \mathrm{~cm}^{-2}$. The most commonly accepted explanation is that the local microstructure of the QWs leads to localisation that prevents carrier diffusion to non-radiative centres. The outstanding questions related to the localisation have concerned the nature of the localisation centres. Probably the most significant step forward in this area was the demonstration by Galtrey et al [2] that the In atoms were distributed randomly in c-plane structures and that previous reports [3,4] of In clustering which was believed to be the cause of carrier localisation could be an artefact of the measurement technique used [5]. Indeed further evidence has emerged that the In atoms in InGaN QWs grown in our laboratory are randomly distributed as has been described by Bennett et al [6] and Tang et al [7]. Along with the suggestion by Graham et al [8] that well width fluctuations could be more effective for localising carriers than previously thought, led to significant reappraisals of the overall energy landscape in InGaN/GaN QWs.

Distinct theoretical approaches by Watson-Parris et al [9] who used an effective mass treatment and Schulz et al [10] who applied an atomistic tight binding model both led to the following overall picture of c-plane $\mathrm{InGaN} / \mathrm{GaN}$ QWs: in the situation where In atoms are randomly distributed they can be very effective at localising holes whereas electrons are more likely to be localised at well width fluctuations. Schulz et al [10] also found that the spatial separation of the electron and hole wave functions due to the presence of the built-in potential 
leads to a relatively weak electron-hole Coulomb interaction so that localised electrons and holes can be considered as independent. The results of both theoretical approaches are supported by the experimental data where it was found that the low temperature photoluminescence (PL) line widths (ignoring phonon effects) were very similar to the calculations of fluctuations in the hole localisation energies. Additionally, one factor which is not explicitly considered in our model is the incorporation of indium into the gallium nitride barrier, which is known to affect the calculated electron and hole energies in continuum models. In general, in our atomistic description the well barrier interface is not as sharp as in a continuum based description. This originates from the fact that the atomic arrangement of In atoms at the well barrier interfaces varies locally. We note that a more realistic indium profile across the quantum well interface was included in our earlier theoretical studies using a modified continuum model [9]. Where the modified continuum model (with the indium penetration into the barrier) and our current atomistic model (without such indium penetration into the barrier) address the same questions, they give very similar results, suggesting that the non-sharp interface is not the key factor influencing the carrier localisationIt should be stressed that in the above we are only referring to the energetically low lying localised electron and hole states. The effects of carrier localisation also manifest themselves in the anomalous temperature dependence of the peak PL energy [11] and the form of the low temperature PL decay curves [12,13]. To gain insight into impact of alloy fluctuations in InGaN/GaN QWs from theory different approaches have been applied. These methods range from localization landscape theory [14][15], modified continuum-based models [9] and atomistic tight-binding calculations [10].

Carrier localisation in semiconductor systems has been widely studied and has proved particularly amenable to optical studies where the photon energy of the excitation is resonant with either specific features in the absorption or excitation spectrum $[16,17]$ or with the 
conventional emission spectrum [18,19]. Somewhat surprisingly the use of resonant spectroscopy has not been widespread in the study of InGaN/GaN QWs but the technique has been used by Satake et al [20], Schmidt et al [21], Graham et al [22] and Hylton et al [23]. Of particular relevance to the work reported here is the previous study [18] where it was observed that at low temperatures as the excitation photon energy was reduced below some critical value the peak PL shifted to lower energy. The onset of this shift was attributed to excitation below a mobility edge [24] that prevented all the localised states being occupied. It should be noted that the observation of a mobility edge in InGaN/GaN QWs has also been reported using pump-probe spectroscopy [25].

In this paper we extend our previous work [22] and study the form of the PL spectrum from a c-plane InGaN/GaN structure at low temperature as a function of excitation energy and correlate the results with atomistic calculations [26] of the ground and excited localised states of electrons and holes. At $\mathrm{T}=12 \mathrm{~K}$ for excitation photon energies above a certain critical energy the form of PL spectrum reflects the random occupation of the localised hole states but for photon energies below this critical energy the form of the spectrum reflects the fact that the holes remain localised in the states in which they are resonantly excited. We interpret the critical energy as being due to a mobility edge whereby the holes cannot scatter to lower lying hole states. The growth of InGaN QWs on sapphire leads to very large defect densities, indeed in the sample reported on here the defect density is $<3-6 \times 10^{8} \mathrm{~cm}^{-2}$. Nevertheless we do not believe these defects influence our conclusions as the work was performed at $12 \mathrm{~K}$ where the effects of defects are negated by localisation, indeed we have demonstrated previously [27] that the internal quantum efficiency in similar structures both at low and high temperatures are independent of defects for densities $<10^{9} \mathrm{~cm}^{-2}$. Also we note that the majority of commercial LEDs are grown on such highly defective substrates and our study is thus relevant to the majority of material grown today. 


\section{Methods}

A 10 period $\operatorname{In}_{0.17} \mathrm{Ga}_{0.83} \mathrm{~N}(2.5 \mathrm{~nm}) / \mathrm{GaN}(7.3 \mathrm{~nm}) \mathrm{QW}$ structure was grown by metal organic vapour-phase epitaxy in a Thomas Swan 6 x 2 inch close-coupled showerhead reactor. The structure was grown on low defect density c-plane sapphire/GaN pseudo-substrates on cplane sapphire with a nominal $0.25^{\circ}$ miscut toward (11-20). The QWs were grown using a Q2T methodology [28] where following the growth of the QW at $756{ }^{\circ} \mathrm{C}$ a $1 \mathrm{~nm}$ thick $\mathrm{GaN}$ QW cap was grown. The temperature was then ramped up to $860{ }^{\circ} \mathrm{C}$ and then the rest of the barrier was grown. The sample was mounted in a closed cycle cryostat at Brewster's angle to minimise the effects of interference fringes [22]. The PL was excited by chopped light from a tuneable dye laser at an excitation power density of $250 \mathrm{Wcm}^{-2}$. The PL was dispersed by a $0.85 \mathrm{~m}$ double grating spectrometer and detected by a GaAs photomultiplier whose output was processed by a lock-in detector. It should be noted that the same results as reported here were also obtained on a sample grown using a $2 \mathrm{~T}$ growth methodology [28] which leads to gross fluctuations in the QW width on a lateral scale of 50 to $100 \mathrm{~nm}$. This implies that the microstructure on a lateral scale smaller than that of the gross well width fluctuations is controlling this aspect of the optical properties.

\section{Results and Discussion}

In figure 1 are shown PL spectra at a temperature of $\mathrm{T}=12 \mathrm{~K}$ when exciting the sample with light of photon energies of $2.973 \mathrm{eV}$ and $2.783 \mathrm{eV}$. The PL spectrum excited by light with the greater photon energy consists of a main recombination with peak energy of $2.774 \mathrm{eV}$ and a weaker feature with peak energy of $2.684 \mathrm{eV}$. These are identified [8] as being due to recombination involving localised electrons and holes $(2.774 \mathrm{eV})$ and $\mathrm{LO}$ phonon assisted $(2.684 \mathrm{eV})$ recombination of the main PL peak. It should be noted that the form of PL spectrum remains unchanged when the excitation photon energy is greater than $2.812 \mathrm{eV}$; we 
refer to spectra taken under this set of conditions as non-resonant PL. When the excitation photon energy was reduced to $2.783 \mathrm{eV}$ in figure 1 the PL spectrum is changed radically. In this particular case the excitation photon energy lies within the non-resonant PL spectrum as indicated by the red arrow, this we refer to as resonant excitation. As discussed by Graham et al [22], following the work by Cohen et al [18] and Permogorov et al [19], the features A $(2.753 \mathrm{eV}), \mathrm{B}(2.690 \mathrm{eV})$ and $\mathrm{C}(2.663 \mathrm{eV})$ are identified as follows. Feature A is ascribed to acoustic phonon assisted absorption and recombination of the directly excited localised electrons and holes at the excitation photon energy, feature B is due to emission from the resonantly excited states which are accompanied by the emission of an LO phonon with feature $\mathrm{C}$ being the $\mathrm{LO}$ phonon replica of feature $\mathrm{A}$.

In figure 2 are shown low temperature PL spectra as the excitation photon energy is moved across the non-resonant PL spectrum. Once the excitation photon energy is less than 2.812 $\mathrm{eV}$, the peaks $\mathrm{A}, \mathrm{B}, \mathrm{C}$ (as defined in figure 1) all move by equal amounts to lower energy reflecting the specific excitation of a subset of the localised electrons and/or holes with the excitation photon energy used. So for excitation photon energies above $2.812 \mathrm{eV}$ the form of the spectra remain unchanged but for excitation photon energies below $2.812 \mathrm{eV}$ the spectral peaks move to lower energy with decreasing excitation photon energy. This behaviour is reflected in the graph in figure 3 . We interpret this behaviour in terms of a mobility edge, which defines the critical energy below which photo excited carriers cannot occupy the full distribution of localised states. To fully understand this behaviour we need to have information on the available energy states for both electrons and holes.

To understand this behaviour, we have applied the atomistic tight-binding model described in refs [10] and [26] to study the electron and hole states of c-plane InGaN/GaN QWs. In this approach we account for well width and random alloy fluctuations. The strain and built-in field variations arising from random alloy fluctuations are treated on a microscopic level by 
using valence force field and local polarization methods. Within this framework we have modelled a c-plane $\operatorname{In}_{0.15} \mathrm{Ga}_{0.85} \mathrm{~N} / \mathrm{GaN}$ QW with a width of approximately $3 \mathrm{~nm}$. We note that whereas our calculations were performed for a QW thickness of 3nm, the measured thickness was $2.5 \mathrm{~nm}$. Since the key point for understanding the data is the detailed description of both ground and localized hole states, the well width is of secondary importance for the conclusions drawn here. Our main focus is on carrier localization and how this feature changes with energy. It is beyond the scope of the present study to match, for instance, transition energies, where the well width will play a role. In the present work, it is more important that quantities such as the FWHM are reasonably described (as they are) by our model, since this gives an indication of the energy distribution of localized states. The calculations have been performed on $\approx 82,000$ atom supercells (equivalent to a system size of $10 \mathrm{~nm} \times 9 \mathrm{~nm} \times 10 \mathrm{~nm}$ ) with periodic boundary conditions. 175 configurations have been used to obtain reliable statistical averages and thus to compare our results with the experimental data. For instance, when calculating the PL emission spectra we find a full width of half maximum (FWHM) value of $\mathrm{FWHM}^{\text {theo }}=84 \mathrm{meV}$ which is in reasonable agreement with the experimental data of $\mathrm{FWHM}^{\mathrm{exp}}=65 \mathrm{meV}$. A detailed discussion of the factors that influence the calculated FWHM is given in our previous work [26] where we noted that the interplay of well width fluctuation and built-in field can affect the variation of the electron ground state energy as a function of the microscopically different configurations. In turn, this can lead to the situation that the calculated FWHM values are larger when compared to the experiment. In general, all this is affected by statistical fluctuations in the size and shape of well width fluctuations and the connected changes in the electronic structure of the system. Given that we have a reasonably good agreement between theory and experiment for the FWHM, and therefore the energy distribution of the localized states, the agreement between theory and experiment is sufficient for the present work, where we are 
interested in factors that influence the observation of the mobility edge. Thus the chosen number of configurations is sufficient to obtain a good description of the energy variation in electron and hole states originating from random alloy fluctuations, which is key to understanding the experimental findings. Central to the existence of a mobility edge is the ability of carriers to transfer from a state of a given energy to another state with a different energy. Moreover, in this context the question is if there is any major difference between electron and hole states. To shed light on this question we have calculated the modulus overlap $\Omega_{n m}^{\lambda}=\Sigma_{i}^{N}\left|\psi_{n, i}^{\lambda}\right|\left|\psi_{m, j}^{\lambda}\right|$, between the states $\psi_{m}^{\lambda}$ and $\psi_{n}^{\lambda}$, for electrons $(\lambda=e)$ and holes $(\lambda=h)$. The index $i$ denotes the lattice site in the atomistic grid. For each of the 175 configurations, the calculation of $\Omega_{n m}^{e}$ has been performed for 5 electron states; for the holes 40 states have been taken into account to calculate $\Omega_{n m}^{h}$. To achieve an energy resolved distribution of $\Omega_{n m}^{\lambda}$ for electrons and holes using the different microscopic configurations, overlaps for given states/energies have been grouped together in energy bins of the width of $30 \mathrm{meV}$ and $20 \mathrm{meV}$ for electrons and holes, respectively. The difference in the bin size accounts for difference in the localization features of electrons and holes. Overlaps in each bin are averaged over the number of elements within the bin. In doing so, and keeping in mind the definition of $\Omega_{n m}^{\lambda}$, only values between 0 (no overlap between states) and 1 (wave functions are normalized) are possible. More details on the modulus overlap calculations are given in ref [26]. The results of our analysis are depicted in Fig. 4 (a) for electrons and in (b) for holes. Here, the zero of energy is taken as the valence band edge of the unstrained bulk GaN. For the sake of discussion, in the following we will use terms such as "conduction band" and "valence band", even though this notation is strictly speaking not correct due to the presence of localized states. Starting with electrons, the bottom left corner of the figure corresponds to the "conduction band" edge which relates to electron states that are affected/localized by well width fluctuations; when approaching the top right corner of figure 
4 (a) one is moving up higher in the "conduction band". Overall, figure 4 (a) clearly shows that there is large overlap between electron states with different energies. This means that once carriers are excited in the "conduction band", electrons can transfer easily from one state to another. Consequently, when electrons are excited above the "conduction band" edge they can easily be transferred to the localized states at the "band" edge. The situation is very different for the holes, as depicted in figure 4 (b). Looking at the "valence band" edge (top right corner of figure 4 (b)), we find strongly localized states, which have very little overlap with states lower in energy, thus deeper in the "valence band". Also, we observe that at lower energies (bottom left corner) the overlaps between hole states are also large and comparable to the numbers observed in our analysis of the electron states (figure 4 (a)).

Thus based on the theoretical results given above, the experimental data in figure 3 can be explained in terms of a mobility edge. Overall it is important to note that the absolute energies calculated here cannot be directly compared to the absolute energies obtained in the experiment. This stems from the fact that for instance the well width and thus the PL peak energy of the non-resonant PL are different between theory and experiment. However, as discussed already above, the FWHM values in theory and experiment are similar, indicating that the spread in electron and hole energies is realistically described by the theory. For the present study of the mobility edge, this feature is key for the theory-experiment comparison. Turning back to figure 3, excitation photon energies above the experimental mobility edge $(2.812 \mathrm{eV})$ results in the occupation of excited electron and hole states, assuming the primary excitation process results in the creation of independent electrons and holes. Such a process would correspond to carriers being excited in the states described by the upper right part of figure 4 (a) (electrons) and bottom left corner of figure 4 (b) (holes). Carriers in these states can, because of the strong wave function overlap between the excited electron and hole states, scatter rapidly on the time scale of the PL recombination down to the localised electron and 
hole states leading to the observed PL spectrum. When lowering the excitation energy, in terms of electron states, one moves from the upper right to the lower left corner. Given that the overlap between electron states with different energies is high and largely unaffected by the change in energy, the electrons can still be transferred to the localized electron states. Thus, we do not expect a significant contribution from the electron system to the mobility edge observed in the experiment. The situation is drastically different for holes. In this case, when lowering the excitation energy, in figure 4 (b) one moves from the bottom left region to the upper right corner. In this case, and in contrast to the electrons, the wave function overlap between the different hole states changes significantly with energy and carriers cannot be easily transferred between different localised states. Thus excitation below the mobility edge results in direct excitation of holes in localised states such that the carriers are not sufficiently mobile at $\mathrm{T}=12 \mathrm{~K}$ to scatter to the other localised states. Hence as the excitation photon energy is reduced the spectrum shifts accordingly as the energy of the states that are directly populated also is progressively reduced. From this analysis we conclude that the mobility edge is mainly determined by hole localisation.

\section{Conclusion}

In summary we have studied the form of the low temperature PL spectrum of a c-plane InGaN/GaN QW structure as a function of excitation energy. For excitation photon energies above $2.812 \mathrm{eV}$ the spectra involve recombination of zero phonon and LO phonon accompanied recombination of localised electrons and holes. For excitation photon energies below $2.812 \mathrm{eV}$ the form of the spectra are radically changed consisting mainly of LO and acoustic phonon assisted recombination of resonantly excited localised states. The peak energies of both these features shift to progressively lower photon energies as the excitation photon energy is reduced. 
This behaviour is consistent with the existence of a mobility edge whereby for excitation below $2.812 \mathrm{eV}$ localised carriers are not free to redistribute across the available localised states. This interpretation is reinforced by a comparison with the results from an atomistic tight-binding model. The results of these calculations show that the wave function overlap of the lower energy localised hole states with other hole states is low whereas the electronelectron wave function overlap is largely independent of energy. On this basis we attribute the mobility edge to the strong localisation of the lowest lying holes.

\section{Acknowledgements}

This work was carried out with the support of the United Kingdom Engineering and Physical Sciences Research Council under grant Nos. EP/M010589/1, EP/I012591/1, EP/M010627/1 and EP/H049533/1 and the Science Foundation Ireland (SFI) under Project No. 13/SIRG/2210. The data associated with the paper are openly available from http://dx.doi.org/10.17632/tdzkmm5gw3.2 


\section{References}

[1] http://www.nobelprize.org/nobel_prizes/physics/laureates/2014/.

[2] Galtrey M. J, Oliver R. A, Kappers M.J, Humphreys C. J, Clifton P, Larson D, Saxey D and Cerezo A 2008, J. Appl. Phys. 104, 013524

[3] Chichibu S, Wada K and S. Nakamura 1997 Appl. Phys. Letts., 71, 2346

[4] Narukawa Y, Kawakami Y, Fujita S, S Fujita and Nakamura S 1997 Phys. Rev B, 55, R1938

[5] Smeeton T M, Kappers M J, Barnard J S, Vickers M E and Humphreys C J 2003 Appl. Phys. Letts., 83, 5419

[6] Bennett S E, Saxey D W, Kappers M J, Barnard J S, Humphreys C J, Smith G D W and Oliver R A, 2011, Appl. Phys. Lett., 99, 21906

[7] Tang F, Zhu T, Oehler F, Fu W Y, Griffiths J T, Massabuau F C-P, Kappers M J, Martin T L, Bagot P A J, Moody M P and Oliver R A, 2015 Appl. Phys. Lett., 106, 72104

[8] Graham D M,Soltani-Vala A, Dawson P, Godfrey M J, Smeeton T M, Barnard J S, Kappers M J, Humphreys C J and Thrush E J, 2005 J. Appl. Phys., 97, 103508

[9] Watson-Parris D, Godfrey M J, Dawson P, Oliver R A, Galtrey M J, Kappers M J and Humphreys C J 2011 Phys. Rev. B, 83, 115321.

[10] Schulz S, Caro M A, Coughlan C and O'Reilly E P 2015 Phys. Rev. B, 91, 035439

[11] Eliseev P G, Perlin P, Lee J and Osinski M 1997 Appl. Phys. Letts.,71, 569

[12] Davidson J A, Dawson P, Wang T, Sugahara T, Orton J W and Sakai S 2000 Semicon. Sci. Tech., 15, 497

[13] Morel A, LefebvreP, Kalliakos S, Taliercio T, Bretagnon T and Gil B, 2003, Phys. Rev. $\mathrm{B}, \mathbf{6 8}, 045331$

[14] Filoche M, Piccardo M, Wu Y-R, Li C-K, Weisbuch C and Mayboroda S, 2017, Phys. Rev B, 95, 144204

[15] Piccardo M, Li C-K, Wu Y-R, Speck J S, Bonef B, Farrell R M, Filoche M, Martinelli, Peretti J and Weisbuch C, 2017, Phys. Rev. B 95, 144205

[16] Brener I, Olszakier M, Cohen E, Ehrenfreund E, A Ron and Pfeiffer L 1992 Phys Rev B, 46, 7927

[17] Dawson P, Buckle P, Godfrey M J, Roepke W H and Halsall M 1997 Sol Stat. Comms., 101, 477 
[18] Cohen E and Sturge M D 1982 Phys. Rev. B, 25, 3828

[19] Permogorov S, Reznitkii A, Verbin S, Muller G O, Flogel P and Nikiforova M 1982 Phys Stat. Sol. B, 113, 589

[20] Satake A, Masumoto Y, Miyajima T, Asatsuma T, Nakamura F and Ikeda M 1998 Phys. Rev. B, 57, R2041

[21] Schmidt T J, Cho Y H, Bidnyk S, Song J J, Keller S, Mishra U K and Denbaars S P 1999 Proc. SPIE Conf. Physics and Simulation of Optoelectronic Devices VII, 3625, 57

[22] Graham D M, Dawson P, Godfrey M J, Kappers M J and Humphreys C J 2006 Appl. Phys. Letts., 89, 211901

[23] Hylton N P, Dawson P, Kappers M J, McAleese C and Humphreys C J 2007 Phys. Rev. B, 76, 205403

[24] Mott N 1987 J. Phys C, 20, 3075

[25] Schmidt T J, Cho Y H, G H Gainer, Song J J, Keller S, Mishra U K and DenBaars S P 1998 Appl. Phys. Letts., 73, 560

[26] Tanner S P, Caro M A, O'Reilly E P and Schulz S 2016 RSC Advances, 6, 64513

[27] Davies M J , Dawson P, Massabuau F C-P, OehlerF, Oliver R A, Kappers M J, Badcock T J, and Humphreys C J,2014, Phys. Status Solidi C 11, 750

[28] Oliver R A, Massabuau F C-P, Kappers M J, Phillips W A. Thrush E J, Tartan C C, Blenkhorn W E, Badcock T J, Dawson P, Hopkins M A, Allsopp D W E and Humphreys C J 2013, Appl. Phys. Letts., 103, 141114 


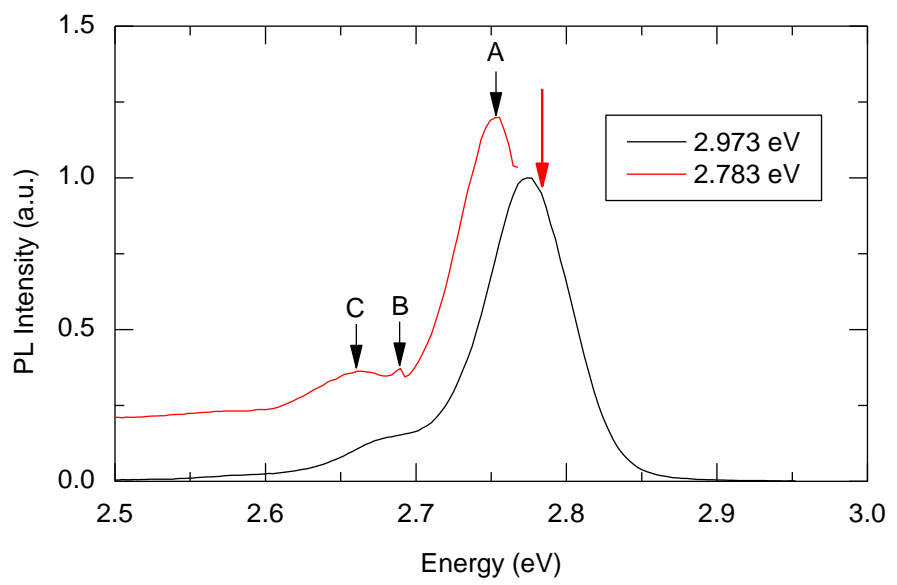

Figure 1. Photoluminescence spectra recorded at $\mathrm{T}=12 \mathrm{~K}$ for excitation photon energies of $2.973 \mathrm{eV}$ and $2.783 \mathrm{eV}$ as indicated. The energy of $2.783 \mathrm{eV}$ is indicated by the red arrow.

The nature of the transitions $\mathrm{A}, \mathrm{B}$ and $\mathrm{C}$ are discussed in detail in the text. 


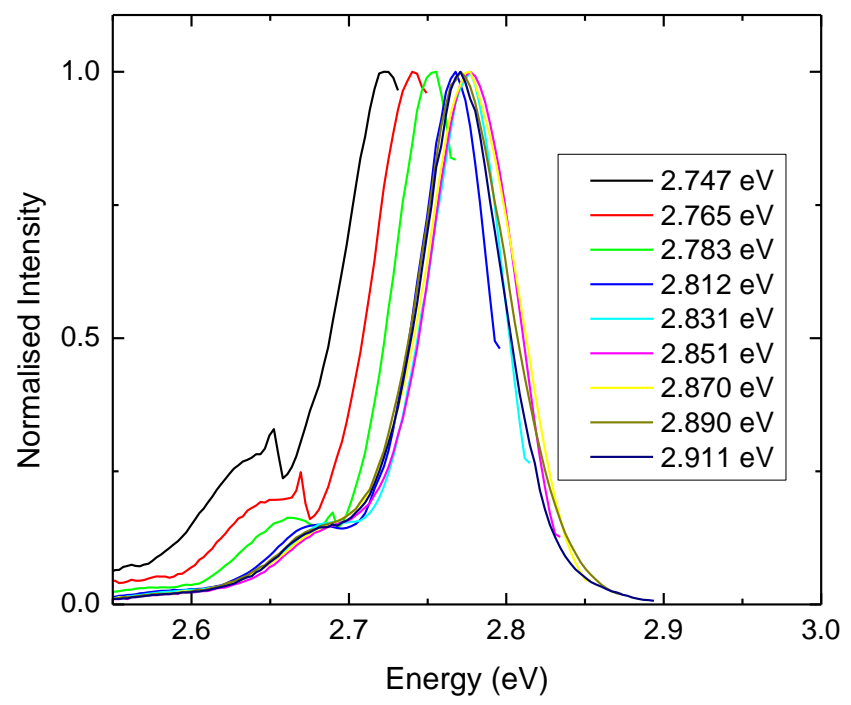

Figure 2. Photoluminescence spectra measured at $\mathrm{T}=12 \mathrm{~K}$ with the excitation photon energies indicated in the figure. 


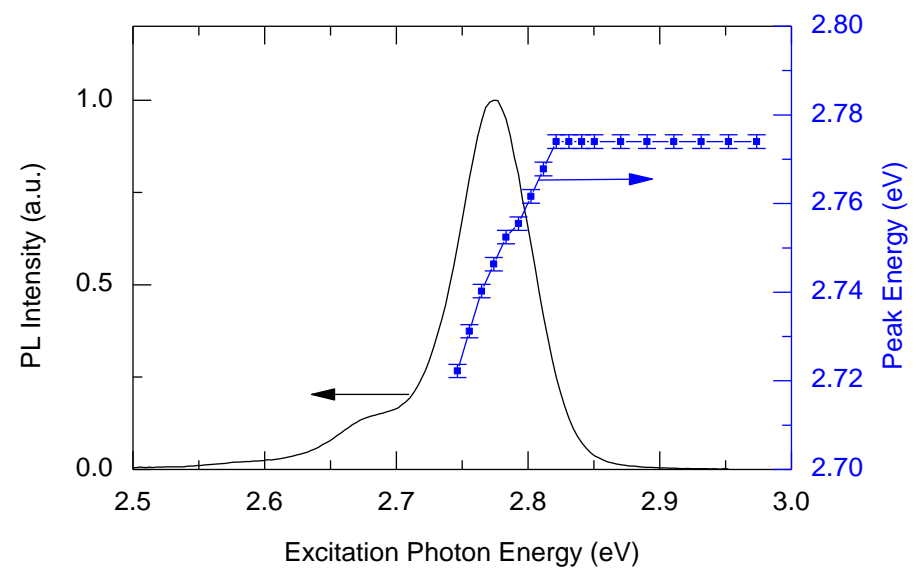

Figure 3. Photoluminescence spectrum (black) measured at $\mathrm{T}=12 \mathrm{~K}$ for excitation photon energy of $2.973 \mathrm{eV}$ alongside a plot (blue circles) of the energy of peak photoluminescence intensity as a function of excitation energy. 
(a)

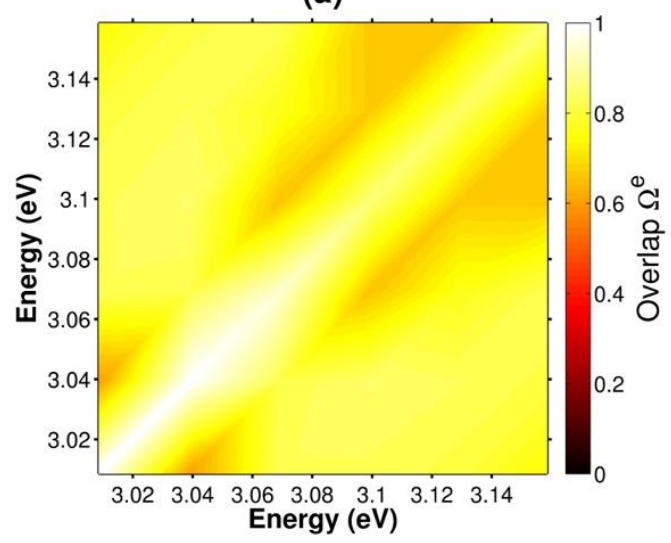

(b)

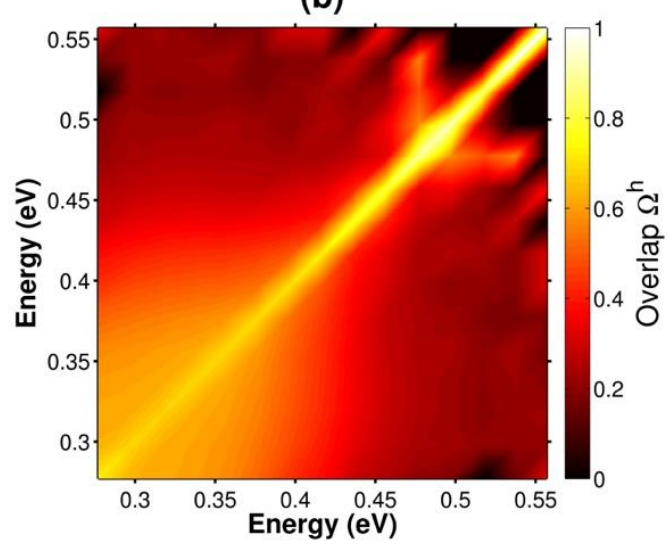

Figure 4 Modulus wave function overlap $\Omega$ for (a) electrons and (b) holes. The valence band edge of unstrained bulk GaN is taken as the zero of energy. Thus the QW "conduction band" edge corresponds to the lower left corner in (a), while the QW "valence band" edge is determined by the upper right corner in (b). 